\title{
A tool for assessing the feasibility of comparative effectiveness research
}

This article was published in the following Dove Press journal:

Comparative Effectiveness Research

29 January 2013

Number of times this article has been viewed

\section{Alexander MWalker ${ }^{1}$ \\ Amanda R Patrick ${ }^{2}$ \\ Michael S Lauer ${ }^{3}$ \\ Mark C Hornbrook ${ }^{4}$ \\ Matthew G Marin ${ }^{5}$ \\ Richard Platt ${ }^{6}$ \\ Véronique L Roger ${ }^{7}$ \\ Paul Stang ${ }^{8}$ \\ Sebastian Schneeweiss ${ }^{2}$ \\ 'World Health Information Science Consultants, Newton, MA; 'Division of Pharmacoepidemiology and Pharmacoeconomics, Brigham and Women's Hospital, Boston, MA; ${ }^{3}$ National Heart, Lung, and Blood Institute, National Institutes of Health, Bethesda, MD; ${ }^{4}$ The Center for Health Research, Kaiser Permanente Northwest, Portland, OR; ${ }^{5}$ Department of Medicine, New Jersey Medical School, Newark, NJ; ${ }^{6}$ Department of Population Medicine, Harvard Pilgrim Health Care Institute and Harvard Medical School, Boston, MA; ${ }^{7}$ Department of Health Sciences Research, Mayo Clinic, Rochester, $\mathrm{MN} ;{ }^{8}$ Johnson and Johnson Pharmaceutical Research and Development, Titusville, NJ, USA}

\section{Video abstract}

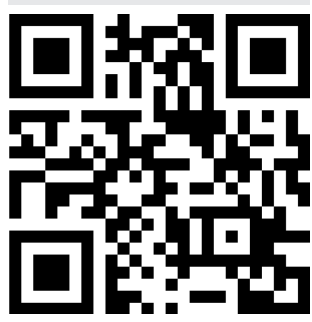

Point your smartphone at the $\mathrm{QR}$ code to the left. If you have a QR code reader the video abstract will appear. Or use: http://dvpres/ WGSkxb
Correspondence: Alexander M Walker 275 Grove Street, Suite 2-400,

Newton, MA 02466, USA

Tel + I 6176635945

Email alec.walker@whiscon.com
Background: Comparative effectiveness research (CER) provides actionable information for health care decision-making. Randomized clinical trials cannot provide the patients, time horizons, or practice settings needed for all required CER. The need for comparative assessments and the infeasibility of conducting randomized clinical trials in all relevant areas is leading researchers and policy makers to non-randomized, retrospective CER. Such studies are possible when rich data exist on large populations receiving alternative therapies that are used as-if interchangeably in clinical practice. This setting we call "empirical equipoise."

Objectives: This study sought to provide a method for the systematic identification of settings it in which it is empirical equipoise that offers promised non-randomized CER.

Methods: We used a standardizing transformation of the propensity score called "preference" to assess pairs of common treatments for uncomplicated community-acquired pneumonia and new-onset heart failure in a population of low-income elderly people in Pennsylvania, for whom we had access to de-identified insurance records. Treatment pairs were considered suitable for CER if at least half of the dispensings of each treatment-pair member fell within a preference range of $30 \%$ to $70 \%$.

Results: Among 3889 community-acquired pneumonia patients, insurance claims histories were sufficiently similar in seven drug pairs to suggest that observational CER might be effective. Relapse appears to have been less common in levofloxacin recipients than in similar patients given other products. In 6035 heart failure patients, metoprolol, carvedilol, and atenolol were employed in patients with similar claims histories, and thus might be suitable for observational CER. The long-acting succinate formulation of metoprolol had lower failure rates in head-to-head comparisons with all other beta-blockers. Both findings are candidates for further investigation. Confounding by unmeasured factors operating in the same manner as the measured covariates would not have produced the apparent superiority of levofloxacin, which was given to people in poorer respiratory health. The baseline covariate distributions of persons starting beta-blockers suggest only that carvedilol recipients were healthier than others.

Conclusion: A straightforward algorithm can identify empirical equipoise, in which prescribers as a group seem evenly divided on the merits of alternative therapies. This is the setting in which CER may be most necessary and is likely to be most accurate. The imbalances identified by propensity models can identify situations in which the results of screening analyses may be biased in the direction of the observed effect.

Keywords: equipoise, observational CER, methodology, community-acquired pneumonia, heart failure

\section{Background}

The Institute of Medicine recently used expert discussion and consensus to identify priorities for comparative effectiveness research (CER). ${ }^{1}$ These were areas in which 
treatments in common use might well have very different outcomes and for which there was little direct information. Perusal of these priority areas suggests that many of these will require a substantial and sophisticated research effort.

We present here an empirical method for identifying possible areas for CER needs. This method might be called the "low-hanging fruit algorithm" because it involves systematically looking for treatment alternatives for which the physician community appears to make very little distinction between which patients receive one treatment and which patients receive the other. This results in largely comparable treatment populations, which in turn can be compared for the occurrence of outcomes of all kinds, with only modest requirements for covariate control.

For example, clinical indifference might arise when: there is a lack of evidence to differentiate two products, there is positive evidence of equivalence, there is an expectation of equivalence because of common pharmacology or membership in a therapeutic class, guidelines give equal weight to therapeutic alternatives, or the net effect of promotion and accessible scientific data has been to leave prescribers without a clear preferred treatment option. The manifestation of clinical indifference in patient data will be that no patient medical or demographic characteristics distinguish recipients of one therapy from those of another. We would like to use the term "empirical equipoise" to identify a setting of strong, observed similarity in the kinds of patients receiving two regimens.

"Equipoise" refers to a balance of opinion in the treating community about what really might be the best treatment for a given class of patients. The existence of equipoise can make a randomized trial ethically justifiable even when no single researcher or clinician is personally in doubt about which might be the best course of treatment; that is, a treating professional can think: "Seeing that others who are equally informed disagree with me, I recognize that we as a treatment community are in a state of equipoise." Empirical equipoise differs from true equipoise in that the balance of prescribers' actions is taken as the measure of preference rather than their opinions.

Under empirical equipoise, individual physicians may have preferences for treatments for particular patients, but the preferences tend to balance out across providers. Not knowing the opinions of prescribers, we cannot say whether there is true equipoise, but we can observe that the prescriber community appears to be acting as if there were equipoise. Empirical equipoise is the practice condition in which comparative observational studies can be pursued with a diminished concern for the "confounding by indication" that plagues non-randomized studies of treatment effect.

The very large bodies of information on medical care and outcomes in governmental and private insurance schemes offer an avenue for prioritizing topics for CER. We can, at least for those settings, identify alternative therapies for which there is empirical equipoise and for which observational, retrospective CER might be most feasible.

\section{Methods}

\section{A prioritization tool}

We propose the following algorithm.

1. Identify an environment with longitudinal health care data for a large population in which CER may be relevant. The data source must credibly capture the critical variables to define the date and nature of the treatment (eg, drug name, days supplied, date prescription filled, dosage, quantity dispensed), likely indications, and relevant outcomes (these might include diagnosis codes, procedure codes, diagnostic test codes, hospital discharge diagnoses).

2. Select treatment indications that have a clear onset that can be dated using the available data, for which there are a variety of treatment options that might be compared, and which are also clearly captured in the available data.

3. Taking all indications pair-wise, perform a regression in which treatment choice with one member of the pair (A vs B) is the dependent variable and the predictors are an exhaustive list of patient characteristics, potential predictors of the outcome, and patterns of care identified before the treatment decision.

4. From the predicted values of the regression, create a preference score ranging from 0 to 1 that has the properties that:

a. patients with preference scores of 0 or 1 receive Treatment A either never or always, respectively

b. intermediate values of the preference score reflect the proportion of patients who would be expected to receive Treatment $\mathrm{A}$ rather than Treatment $\mathrm{B}$, under the circumstance that Treatment A and Treatment B had equal market share

c. (as a consequence of b) patients whose preference score is 0.5 are likely to receive Treatment A or B exactly in proportion to the market shares of Treatments A and B.

5. Accept drug pairs as emerging from empirical equipoise if at least half of the dispensings of each of the drugs are to patients with a preference score of between 0.3 and 0.7 . 


\section{Data source for case studies}

We selected records of patients from the Pennsylvania Pharmacy Assistance Contract for the Elderly (PACE) linked to Medicare Parts A and B claims from 2000 through 2005. To be eligible for PACE at the time, a person's annual income had to be $<$ US $\$ 13,000$ if single and $<$ US $\$ 16,200$ if married. The program reimburses the cost of all prescriptions with a US\$6 co-payment. To our knowledge, there were no formulary restrictions for antibiotics or beta-blockers in the program for this period.

PACE's computerized records include prescription drug name, dosage, quantity dispensed, days supplied, date dispensed, and a code indicating the prescribing physician. Outpatient and inpatient diagnoses, procedure codes, and dates of all inpatient and outpatient services obtained from Medicare claims data were linked to pharmacy dispensing data and to Pennsylvania vital-statistics files. To protect the privacy of subjects and their physicians, all personal identifiers had already been transformed into de-indentified study numbers in the study files to which we had access. We obtained ethics approval from the Brigham and Women's Hospital Institutional Review Board and data use agreements from PACE and the Center for Medicare and Medicaid Services to use their data.

\section{Preference score}

To quantify the degree of overlap in physician choice between two drugs, we employed a propensity score whose components could be identified by an algorithm. There were two motivations for an algorithmic rather than expert-derived score; we wished to: (1) mimic a screening project in which a data source could be reviewed for a large number of possible treatment-pair candidates for CER and (2) take advantage of the rich predictor data and a large number of observations.

The propensity scores for persons receiving Treatment A versus those receiving Treatment $\mathrm{B}$ are the fitted values of the logistic regression derived in comparing persons receiving Treatment A to those receiving B. The components of the propensity score for the screening exercise described here were indicator variables for age in decades on index date (see later in this paper for case-specific definitions of the index date); sex; three-digit International Classification of Diseases, 9th Revision - Clinical Modification (ICD-9) ${ }^{2}$ codes appearing as a principal hospital diagnosis in the periods $1-7$ and $8-270$ days before the index date (retained if found in at least $2 \%$ of the population); three-digit ICD-9 codes appearing in association with a physician outpatient visit in the periods $1-7$ and 8-270 days before the index date (retained if found in at least $2 \%$ of the population); and the following general indicators of health in the 270 days before the index date: number of drugs used (at distinct generic entity level), ${ }^{3}$ number of physician visits, indicator $(\mathrm{Y} / \mathrm{N})$ for hospitalization, indicator $(\mathrm{Y} / \mathrm{N})$ for nursing home admission, and Charlson comorbidity score. ${ }^{4,5}$

We derived a transformation of the propensity score that would be more interpretable as a measure of the preference for one drug or another associated with patient demographic and health characteristics. This preference score was obtained by subtracting the natural logarithm of Treatment A prevalence divided by Treatment B prevalence from the logit of the propensity score, and taking the anti-logit (expit) of the result. In the resulting equation (Equation 1), in the universe of persons receiving either Treatment A or B, $F$ and $S$ are the preference score and propensity score for receiving Treatment A, respectively, and $P$ is the fraction of persons receiving Treatment $\mathrm{A}$ :

$$
\operatorname{In}\left(\frac{F}{1-F}\right)=\operatorname{In}\left(\frac{S}{1-S}\right)-\operatorname{In}\left(\frac{P}{1-P}\right)
$$

To confirm this works in an extreme case, imagine that no patient predictors differentiate use of Treatment $\mathrm{A}$ from use of Treatment B, and that the ratio of prevalence of Treatment $\mathrm{A}$ to Treatment $\mathrm{B}$ is $1 / 9$ (ie, Treatment $\mathrm{A}$ has a $10 \%$ share of the treatments that are either A or B). All the coefficients of the propensity score would be zero and all subjects would have a propensity score of 0.10 . The intercept of the regression equation would be $\ln [0.1 /(1-0.1)]=-2.20$. The procedure for deriving a preference score would subtract $\ln (1 / 9)=-2.20$ from the logit of the propensity score of each subject, making each logit preference score zero. A zero logit preference corresponds to a $50 \%$ preference, since $\operatorname{logit}(0.5)=0$. Everybody would correctly get a preference score of 0.50 because nobody would possess a characteristic that made treatment with A more or less likely than use of Treatment A overall.

\section{Case study I: community-acquired pneumonia (CAP)}

The Infectious Disease Society of North America guidelines identify persons without chronic disease predisposing to drug-resistant Streptococcus pneumoniae infection and without recent antibiotic therapy as a population for whom optimal treatment of CAP involves a relatively small number of options. ${ }^{6}$ This screening exercise aims to identify all commonly used treatments in this setting and to compare them for preliminary evidence of disparities in outcome. 


\section{Methods}

\section{Study subjects}

PACE recipients were included in this analysis if they had all three of the following findings in their insurance files:

- an outpatient physician visit bearing ICD-9 codes 482.9 (bacterial pneumonia unspecified), 485 (bronchopneumonia, organism unspecified), or 486 (pneumonia, organism unspecified) following at least 270 days of uninterrupted recorded data in PACE

- a radiologic exam of the chest up to 3 days before or 2 days after the day of diagnosis. These are marked in insurance files by CPT- 4 codes 71010 (single view, frontal), 71015 (stereo, frontal), and 71021 (two views, frontal and lateral) as well as ICD-9 87.44 (routine chest X-ray, so described, including X-ray of chest not otherwise specified)

- a dispensing from 3 days before to 3 days after the day of diagnosis of single antibiotics indicated or used for CAP. We began with a broad list of all systemically absorbed antibiotics, but restricted analysis to those given to at least $5 \%$ of the CAP patients. The few individuals who received multiple antibiotics were not included in the analysis.

Individuals who qualified for selection on multiple occasions were retained only the first time they qualified for inclusion in the study. The date of initiation of antibiotic treatment was taken as Day 0. Persons meeting all of the just-outlined criteria were excluded from further study if they had:

- an insurance claim during the 270 days prior to Day 0 bearing ICD-9 code 042.x (human immunodeficiency virus infection)

- any dispensing in the preceding 3 months of insulin, or any of the antibiotics used to define treatment groups (as outlined previously)

- a discharge from an inpatient hospital stay with any diagnosis on Day -90 through Day 0

- an ICD-9 code on Day -30 through Day 0 of 480 (viral pneumonia); 482 (pneumonia due to Klebsiella pneumoniae, Pseudomonas, Haemophilus influenzae, Staphylococcus, other specified bacteria); 483 (pneumonia due to Mycoplasma pneumonia, Chlamydia, other specified organism); 484 (pneumonia in cytomegalic inclusion disease, whooping cough, anthrax, aspergillosis, other systemic mycoses, other infectious diseases classified elsewhere); 487 (influenza); 488 (influenza due to identified avian influenza virus); pneumonia in other conditions: 518.3 (allergic or eosinophilic), 507 (aspiration), 770.0 (congenital), 514 (passive), 390 (rheumatic), 997.31 (ventilator associated).

\section{Treatment failure}

We identified treatment failure as the earliest (if any) occurrence within 30 days after Day 0 of: hospitalization with either a principal discharge diagnosis of ICD-9 481 (pneumococcal pneumonia), 482.9 (bacterial pneumonia unspecified), 485 (bronchopneumonia, organism unspecified), 486 (pneumonia, organism unspecified, 510 (empyema), or 513.0 (abscess of lung) or a new dispensing of any antibiotic. Note that the hospitalization codes for treatment failure do not include 480 (viral pneumonia), 487 (influenza), or 488 (avian influenza).

\section{Results}

There were 30,291 cases of apparent CAP. From these, to identify uncomplicated cases as explained in the earlier section "Study subjects," we serially excluded: 989 patients with less than 270 days of baseline information, 13 with human immunodeficiency virus, 15,447 with recent antibiotic use, and 8611 with codes indicating a viral pneumonia or pneumonia due to an agent other than S. pneumoniae. This left 5231 that met the screening criteria for uncomplicated CAP. The antibiotics used by at least $5 \%$ of the uncomplicated cases were: azithromycin (28\%), levofloxacin (27\%), clarithromycin (7\%), moxifloxacin (6\%), and amoxicillin $(5 \%)$. Agents not included, but with prevalence of use greater than $1 \%$ were: amoxicillin/clavulanate (4\%), gatifloxacin $(3 \%)$, ciprofloxacin $(3 \%)$, cefuroxime $(3 \%)$, doxycycline $(3 \%)$, cephalexin $(2 \%)$, sulfamethoxazole/trimethoprim $(2 \%)$, and erythromycin (1\%).

Table 1 shows the prevalence of demographic characteristics and the 25 most commonly noted baseline diagnoses by drug. Consistent with expectations for PACE recipients with pneumonia, this was an elderly population (mean age 82 years) of mostly women (80\%). Despite the substantial filter for good health imposed by removing individuals with hospitalizations in the preceding 90 days, the population had high prevalence of previous respiratory and cardiac disease, as a well as a wide range of other chronic ailments. The antibiotic levofloxacin showed the highest prevalence of all common categories of respiratory conditions in the baseline 270 days. These included: respiratory and chest symptoms, pneumonia, and chronic obstructive pulmonary disease. Although receipt of isoniazid or pyrazinamide was not an exclusion criterion, none of the patients counted in Table 1 had received either of these drugs in the preceding 270 days.

Table 2 shows all the possible treatment comparisons, sorted by the proportion with preference values in the range 
Table I Demographics, health care utilization, and outpatient diagnoses present in at least $10 \%$ of patients in the 270 days before initiation of treatment for community-acquired pneumonia

\begin{tabular}{|c|c|c|c|c|c|c|}
\hline \multicolumn{2}{|c|}{ Patient demographics } & Amoxicillin & Azithromycin & Clarithromycin & Levofloxacin & Moxifloxacin \\
\hline \multicolumn{2}{|c|}{ Patients, $\mathrm{n}$} & 269 & 1468 & 369 & 1407 & 320 \\
\hline \multicolumn{2}{|c|}{ Age in years, mean } & 82 & 82 & 81 & 82 & 81 \\
\hline \multicolumn{2}{|c|}{ Female, $\%$} & 81 & 82 & 79 & 78 & 76 \\
\hline \multicolumn{2}{|c|}{ Prior nursing home admission, \% } & 5 & 4 & 3 & 8 & 4 \\
\hline \multicolumn{2}{|c|}{ Prior hospitalization, \% } & 19 & 19 & 15 & 22 & 17 \\
\hline \multicolumn{2}{|c|}{ Distinct drugs dispensed, mean } & 8 & 9 & 8 & 9 & 9 \\
\hline \multicolumn{2}{|c|}{ Physician visits, mean } & 6 & 7 & 6 & 7 & 7 \\
\hline \multicolumn{2}{|c|}{ Comorbidity score, mean } & 2 & 2 & 2 & 2 & 2 \\
\hline \multicolumn{7}{|c|}{ Outpatient diagnoses, $\%$} \\
\hline \multicolumn{7}{|c|}{ ICD-9 $\quad I-7$ days before first dispensing } \\
\hline 486 & Pneumonia, organism unspecified & 6 & 16 & 13 & 23 & 13 \\
\hline \multirow[t]{2}{*}{786} & Symptoms involving respiratory system chest & 6 & 16 & 12 & 20 & 11 \\
\hline & $8-270$ days before first dispensing & & & & & \\
\hline 401 & Essential hypertension & 53 & 55 & 53 & 51 & 54 \\
\hline 110 & Dermatophytosis & 34 & 28 & 22 & 29 & 27 \\
\hline 272 & Disorders of lipid metabolism & 22 & 28 & 26 & 26 & 33 \\
\hline 414 & Other chronic ischemic heart disease & 27 & 28 & 19 & 27 & 26 \\
\hline 786 & Symptoms of respiratory system and chest & 24 & 25 & 22 & 29 & 22 \\
\hline V04 & Need for prophylactic vaccination & 24 & 24 & 27 & 25 & 24 \\
\hline 780 & General symptoms & 18 & 22 & 18 & 21 & 21 \\
\hline 715 & Osteoarthrosis & 25 & 20 & 18 & 22 & 21 \\
\hline 729 & Other disorders of soft tissues & 21 & 20 & 16 & 21 & 22 \\
\hline 250 & Diabetes mellitus & 22 & 20 & 20 & 19 & 23 \\
\hline 427 & Cardiac dysrhythmias & 19 & 20 & 18 & 20 & 16 \\
\hline 366 & Cataract & 19 & 18 & 18 & 20 & 14 \\
\hline 496 & Chronic airway obstruction NEC & 12 & 18 & 15 & 20 & 20 \\
\hline 443 & Other peripheral vascular disease & 19 & 18 & 13 & 19 & 16 \\
\hline 719 & Other and unspecified disorders of joint & 17 & 17 & 12 & 19 & 19 \\
\hline 428 & Heart failure & 19 & 17 & 14 & 16 & 15 \\
\hline 244 & Acquired hypothyroidism & 10 & 15 & 9 & 15 & 13 \\
\hline 733 & Other disorders of bone and cartilage & 12 & 13 & 9 & 14 & 19 \\
\hline 362 & Other retinal disorder & 15 & 13 & 10 & 13 & 14 \\
\hline 724 & Other and unspecified disorders of back & 14 & 13 & 13 & 13 & 10 \\
\hline 365 & Glaucoma & 9 & 12 & 8 & 11 & 11 \\
\hline V58 & Encounter for unspecified care & 10 & 10 & 9 & 11 & 12 \\
\hline 285 & Other and unspecified anemias & 7 & 11 & 9 & 11 & 9 \\
\hline
\end{tabular}

Note: Data from Pennsylvania Pharmacy Assistance Contract for the Elderly (PACE), 2000-2005.

Abbreviations: ICD-9, International Classification of Diseases, 9th Revision - Clinical Modification ${ }^{10}$; NEC, not elsewhere classified.

of 0.3 to 0.7 . The high proportions in the specified range for the first pair listed indicate that azithromycin and levofloxacin were given to the most similar groups of patients, while those in the last pair (amoxicillin and moxifloxacin) were given to the least similar groups. Figure 1 shows smoothed preference score distributions for a similar pair of treatment alternatives (azithromycin and levofloxacin) and a dissimilar pair (clarithromycin and moxifloxacin).

Table 3 presents the risk of presumed treatment failure between pairs of drugs with substantially similar usage, defined as having at least half the dispensings of both agents administered to patients who themselves were within a preference range of 0.3 to 0.7 . Crude and preference score-adjusted effect estimates are very similar, and point to a lower risk of treatment failure in users of levofloxacin compared with each of the other drugs.

\section{Case study 2: heart failure}

The American College of Cardiology, the American Heart Association, and the International Society for Heart and Lung Transplantation have provided guidelines calling for use of beta-blockers in patients with heart failure and depressed left-ventricular systolic function. ${ }^{6}$ The guidelines identify three beta-blockers that have been shown to improve survival: bisoprolol, sustained-release metoprolol, and carvedilol. Other beta-blockers have either not been tested or failed to improve survival in randomized trials. There has been only one large-scale trial comparing different beta-blockers, 
Table 2 Numbers of patients and preference overlap for antibiotic pairs taken by at least $5 \%$ of patients with communityacquired pneumonia

\begin{tabular}{llll}
\hline Antibiotic pair & Patients, $\mathbf{n}$ & \multicolumn{2}{c}{$\mathbf{0 . 3}$ s preference $\leq \mathbf{0 . 7}$} \\
\cline { 3 - 4 } & & $\%$ & $\mathbf{N}$ \\
\hline Azithromycin & 1468 & 85 & 1254 \\
Levofloxacin & 1407 & 82 & 1159 \\
Azithromycin & 1468 & 61 & 889 \\
Clarithromycin & 369 & 66 & 244 \\
Azithromycin & 1468 & 59 & 867 \\
Moxifloxacin & 320 & 56 & 178 \\
Clarithromycin & 369 & 60 & 222 \\
Levofloxacin & 1407 & 55 & 779 \\
Amoxicillin & 269 & 60 & 162 \\
Levofloxacin & 1407 & 57 & 799 \\
Amoxicillin & 269 & 55 & 148 \\
Azithromycin & 1468 & 50 & 740 \\
Levofloxacin & 1407 & 53 & 752 \\
Moxifloxacin & 320 & 60 & 193 \\
Clarithromycin & 369 & 39 & 145 \\
Moxifloxacin & 320 & 42 & 133 \\
Amoxicillin & 269 & 43 & 116 \\
Clarithromycin & 369 & 43 & 159 \\
Amoxicillin & 269 & 39 & 104 \\
Moxifloxacin & 320 & 42 & 113 \\
\hline Note Dat & &
\end{tabular}

Note: Data from the Pennsylvania Pharmacy Assistance Contract for the Elderly program, 2000-2005..$^{10}$

but it failed to use maximal doses for both agents. ${ }^{7}$ Recent observational studies suggest no significant difference between sustained-release metoprolol and carvedilol or between "evidenced-based beta-blockers" (carvedilol, metoprolol succinate, and bisoprolol fumarate) and others among persons with established heart failure and a recent hospitalization..$^{8,9}$

This screening exercise aimed to identify all commonly used beta-blockers in the setting of apparently new-onset heart failure and to compare them for preliminary evidence of disparities in outcome, doing this in a way that would be readily replicable in other populations.

\section{Methods}

\section{Study subjects}

PACE recipients were included in this analysis if they had all three of the following findings in their insurance files within a 28-day period:

- a dispensing of any of the following orally administered beta-blockers: acebutolol, atenolol, metoprolol tartrate, metoprolol succinate, carvedilol, bisoprolol, propranolol, sotalol, labetalol, pindolol, nadolol, timolol, or nebivolol

- an outpatient physician visit or principal hospital discharge diagnosis bearing the first instance in the patient record of ICD-9 code for heart failure (428) other than pure diastolic failure (428.3), following at least 365 days of uninterrupted recorded data in PACE

- evidence that ejection fraction was measured, as attested by the performance of echocardiography, gated single photon emission computed tomography myocardial perfusion imaging, multi-gated acquisition scan, contrast left ventriculography, cardiac magnetic resonance imaging, or fast scan cardiac computed tomography.

The date of the last occurring of these three events was taken as Day 0. Persons previously hospitalized and discharged with a diagnosis of heart failure were excluded (heart failure diagnoses were ICD-9 codes 398.91, 402.01, 402.11, 402.91, 404.01, 404.03, 404.11, 404.13, 404.91, 404.93, 428.0, 428.1, 428.20, 428.21, 428.22, 428.23, 428.30, 428.31, $428.32,428.33,428.40,428.41,428.42,428.43$, or 428.9 .)

\section{Treatment failure}

We identified treatment failure as either hospitalization by Day 60 with a principal discharge diagnosis of heart failure or death from any cause.

\section{Results}

There were 5247 cases of new-onset heart failure. The betablockers that comprised at least $5 \%$ of the initial prescriptions

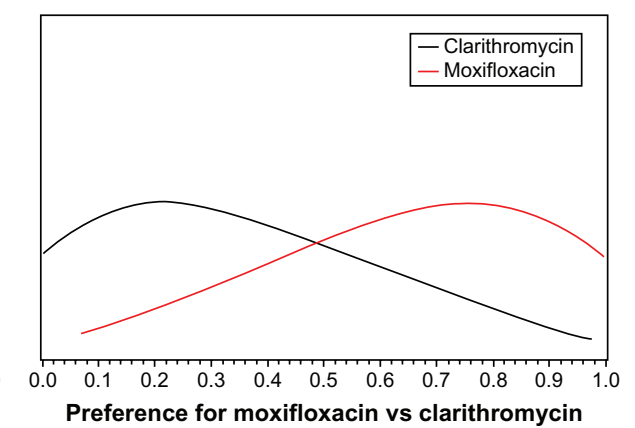

Figure I Preference score distributions.

Notes: Preference distributions for a pair of antibiotics given to very similar patients (left) and for a pair given to substantially different patient populations. (See Table I for salient differences). 
Table 3 Relative risk of presumed treatment failure in community-acquired pneumonia elders

\begin{tabular}{llllll}
\hline $\begin{array}{l}\text { Antibiotic } \\
\text { pair }\end{array}$ & $\begin{array}{l}\text { Patients, } \\
\mathbf{n}\end{array}$ & $\begin{array}{l}\text { Failures, } \\
\mathbf{n}\end{array}$ & $\begin{array}{l}\text { Relative } \\
\text { risk, } \\
\text { crude }\end{array}$ & $\begin{array}{l}\text { PS- } \\
\text { adjusted } \\
\text { odds ratio }\end{array}$ \\
\hline Levofloxacin & 1159 & 337 & 29 & 0.72 & 0.62 \\
Azithromycin & 1254 & 508 & $4 I$ & & $(0.52-0.74)$ \\
Clarithromycin & 244 & 93 & 38 & 0.99 & 0.96 \\
Azithromycin & 889 & 342 & 38 & & $(0.7 I-1.30)$ \\
Azithromycin & 740 & 304 & $4 I$ & 0.67 & 0.42 \\
Amoxicillin & 148 & 91 & $6 I$ & & $(0.29-0.61)$ \\
Moxifloxacin & 178 & 60 & 34 & 0.83 & 0.70 \\
Azithromycin & 867 & 352 & $4 I$ & & $(0.49-0.99)$ \\
Levofloxacin & 779 & 222 & 28 & 0.71 & 0.59 \\
Clarithromycin & 222 & 89 & 40 & & $(0.43-0.8 I)$ \\
Levofloxacin & 799 & 258 & 32 & 0.54 & 0.32 \\
Amoxicillin & 162 & 96 & 59 & & $(0.22-0.45)$ \\
Levofloxacin & 752 & 225 & 30 & 0.95 & 0.95 \\
Moxifloxacin & 193 & $6 I$ & 32 & & $(0.67-1.34)$ \\
\hline Note: Dat from & &
\end{tabular}

Note: Data from the Pennsylvania Pharmacy Assistance Contract for the Elderly program, 2000-2005. ${ }^{10}$

Abbreviation: PS, preference score.

were metoprolol succinate (the extended-release formulation of metoprolol in the USA), metoprolol tartrate, carvedilol, and atenolol. Carvedilol recipients included somewhat fewer women and fewer prior nursing home residents than patients who received the other beta-blockers. Further, fewer carvedilol recipients had had care associated with any of the common diagnoses shown Table 4, which includes all diagnoses seen in at least $10 \%$ of the population. Table 5 , which shows that all the drug pairs met our criterion for comparability, nonetheless confirms the impression that carvedilol usage differed modestly from that of the other agents: all the most distinct drug pairs (those with a lower preference overlap) involved carvedilol.

Table 6 presents the risk of presumed treatment failure between patients in the overlapping range of preferences between the two products. Pairs of beta-blockers are ordered in Table 6 as in Table 5. Metoprolol succinate, the extendedrelease formulation of metoprolol, appears to carry a lower risk of treatment failure than the other three products, and metoprolol tartrate a lower risk than carvedilol.

\section{Discussion}

Empirical equipoise is at the heart of this tool for identifying feasible areas for CER. We propose a systematic method for finding pairs of treatments in which identified patient characteristics do not seem to play an important role in determining which patient receives which therapy. If patient characteristics are not determinative, it follows that selection has been driven by administrative factors (such as varying formularies and copayments or institutional treatment guidelines) and physician preferences (whether these are based on empirical evidence or sales persuasion is irrelevant for the present purpose). To the extent that non-patient determinants of therapy are also not determinative of outcome, they can play a role analogous to randomization in forming comparative groups. Randomization and patient-unrelated treatment assignment share the key characteristic of not resulting in any systematic expectation of greater success of one treatment over the other.

An attractive aspect of the comparative analysis of patients with overlapping preference scores is that the balance in the compared populations is a baseline characteristic. If the compared agents have different patterns of effects (not just treatment success but side effects, for example, or even side effects associated with particular patient attributes) the baseline balance in patient attributes gives confidence that known factors do not confound the drug-to-drug comparison.

The suitability for CER of treatments whose assignment appears to be unrelated to patient attributes depends on the balance and nature of unmeasured predictors of treatment choice. Preference, like the propensity score on which it is based, incorporates only those patient attributes that are known in the data system. Factors such as patient preference, patient experience with other medications, and even the patient-physician relationship will affect the choice of medications, particularly of those that require careful surveillance. By definition, we do not directly know about unmeasured predictors, but the clinical setting described measured predictors can be strongly suggestive. In the example presented of CAP treatment in elders who had not been recently hospitalized, the higher prevalence of respiratory illness in the histories of patients who received levofloxacin, rather than azithromycin, suggests that there is a systematic tendency for physicians to prefer levofloxacin in the presence of such a history. The measured predictors pointed to the levofloxacin patients being, if anything, worse off at baseline, and it seems at least plausible that physicians might similarly have preferred levofloxacin to azithromycin in patients with other unmeasured baseline markers of poor prognosis. In the example of initial treatment for heart failure, the somewhat healthier status of the carvedilol recipients militates against residual confounding as the explanation for its apparent inferiority to both formulations of metoprolol. There is little in the summary patient characteristics to suggest a tendency for persons with less severe underlying disease to have received metoprolol succinate than other products, so again an explanation by confounding seems not 
Table 4 Demographics, health care utilization, recent inpatient diagnoses and outpatient diagnoses present in at least $10 \%$ of patients in the 270 days before initiation of treatment for heart failure

\begin{tabular}{|c|c|c|c|c|c|}
\hline \multicolumn{2}{|c|}{ Patient demographics } & $\begin{array}{l}\text { Atenolol } \\
\left(\text { Tenormin }^{\circledR}\right)\end{array}$ & $\begin{array}{l}\text { Carvedilol } \\
\left.\text { (Coreg }^{\circledR}\right)\end{array}$ & $\begin{array}{l}\text { Metoprolol succinate } \\
\left(\text { Toprol-XL }{ }^{\circledast} \text { ) }\right.\end{array}$ & $\begin{array}{l}\text { Metoprolol tartrate } \\
\text { (Lopressor }^{\circledR} \text { ) }\end{array}$ \\
\hline \multicolumn{2}{|c|}{ Patients, $\mathrm{n}$} & 619 & 907 & 1164 & 2303 \\
\hline \multicolumn{2}{|c|}{ Age in years, mean } & 82 & 81 & 81 & 82 \\
\hline \multicolumn{2}{|c|}{ Female, \% } & 82 & 72 & 78 & 80 \\
\hline \multicolumn{2}{|c|}{ Prior nursing home admission, $\%$} & 36 & 26 & 34 & 40 \\
\hline \multicolumn{2}{|c|}{ Prior hospitalization, \% } & 4 & 3 & 3 & 4 \\
\hline \multicolumn{2}{|c|}{ Distinct drugs dispensed, mean } & 7 & 7 & 7 & 7 \\
\hline \multicolumn{2}{|c|}{ Physician visits, mean } & 4 & 4 & 4 & 4 \\
\hline \multicolumn{2}{|c|}{ Comorbidity score, mean } & 2 & 2 & 2 & 2 \\
\hline \multicolumn{6}{|c|}{ Inpatient diagnoses } \\
\hline \multicolumn{6}{|c|}{ ICD-9 I-7 days before day 0} \\
\hline 410 & Acute myocardial infarction & 4 & 3 & 7 & 8 \\
\hline 414 & Other forms of chronic ischemic heart disease & 3 & 0 & 2 & 3 \\
\hline 427 & Cardiac dysrhythmias & 5 & 2 & 3 & 3 \\
\hline \multicolumn{6}{|c|}{ Outpatient diagnoses } \\
\hline \multicolumn{6}{|c|}{ I-7 days before day 0} \\
\hline 786 & Symptoms of respiratory system and chest & 22 & 17 & 28 & 26 \\
\hline 401 & Essential hypertension & 19 & 10 & 17 & 17 \\
\hline 427 & Cardiac dysrhythmias & 15 & 10 & 15 & 14 \\
\hline 414 & Other forms of chronic ischemic heart disease & 13 & 9 & 13 & 16 \\
\hline \multirow[t]{2}{*}{780} & General symptoms & 10 & 7 & 11 & 12 \\
\hline & 8-270 days before first dispensing & & & & \\
\hline 401 & Essential hypertension & 56 & 51 & 55 & 55 \\
\hline 250 & Diabetes mellitus & 30 & 33 & 29 & 31 \\
\hline 786 & Symptoms of respiratory system and chest & 28 & 26 & 29 & 28 \\
\hline 272 & Disorders of lipoid metabolism & 28 & 27 & 30 & 27 \\
\hline 110 & Dermatophytosis & 29 & 25 & 28 & 28 \\
\hline 414 & Other forms of chronic ischemic heart disease & 23 & 27 & 24 & 26 \\
\hline 715 & Osteoarthrosis & 25 & 21 & 24 & 24 \\
\hline 780 & General symptoms & 23 & 20 & 23 & 22 \\
\hline V04 & Need for prophylactic vaccination & 22 & 20 & 19 & 21 \\
\hline 729 & Other disorders of soft tissues & 19 & 20 & 18 & 20 \\
\hline 719 & Other and unspecified disorders of joint & 22 & 17 & 18 & 19 \\
\hline 443 & Other peripheral vascular disease & 18 & 17 & 20 & 19 \\
\hline 427 & Cardiac dysrhythmias & 18 & 19 & 21 & 17 \\
\hline 366 & Cataract & 20 & 15 & 20 & 19 \\
\hline 362 & Other retinal disorder & 17 & 15 & 16 & 17 \\
\hline 724 & Other and unspecified disorders of back & 14 & 10 & 15 & 14 \\
\hline 244 & Acquired hypothyroidism & 13 & 12 & 12 & 12 \\
\hline 365 & Glaucoma & 13 & 9 & 14 & 12 \\
\hline 429 & III-defined heart disease & 12 & 11 & 13 & 12 \\
\hline 285 & Other and unspecified anemias & 10 & 12 & 11 & 13 \\
\hline 733 & Other disorders of bone and cartilage & 9 & 9 & 12 & II \\
\hline 789 & Other symptoms of abdomen and pelvis & 12 & 10 & 9 & 12 \\
\hline 496 & Chronic airway obstruction NEC & 9 & 12 & 9 & 12 \\
\hline 782 & Symptoms involving skin and integument & 12 & 10 & 11 & 10 \\
\hline V58 & Encounter for unspecified care & 9 & 12 & 10 & 10 \\
\hline
\end{tabular}

Note: Data from Pennsylvania Pharmacy Assistance Contract for the Elderly (PACE), 2000-2005.

Abbreviations: ICD-9, International Classification of Diseases, 9th Revision - Clinical Modification ${ }^{10}$; NEC, not elsewhere classified.

well supported. In both examples, the distinctions identified in the screening procedure deserve to be the object of more rigorous investigation.

Both examples considered here have to do with initial therapy for an acute or evolving condition. For many second-line therapies and for treatments whose implementation is not related to an identifiable clinical episode, the weight of unmeasured determinants of treatment initiation may be too great to derive presumptive equipoise from a similarity in measured characteristics. 
Table 5 Pairs of beta-blockers for which $>50 \%$ of patients on each drug have a preference score between 0.3 and 0.7

\begin{tabular}{lllll}
\hline Beta-blocker pair & \multicolumn{2}{l}{ Total } & & \multicolumn{2}{c}{$\mathbf{0 . 3} \leq$ preference $\leq \mathbf{0 . 7}$} \\
\cline { 2 - 2 } \cline { 5 - 6 } & Patients, $\mathbf{n}$ & & $\%$ & $\mathbf{N}$ \\
\hline Metoprolol tartrate & 2303 & & $83 \%$ & 1917 \\
Atenolol & 619 & & $85 \%$ & 527 \\
Metoprolol succinate & 1164 & & $86 \%$ & 998 \\
Metoprolol tartrate & 2303 & & $86 \%$ & 1983 \\
Metoprolol succinate & 1164 & & $73 \%$ & 850 \\
Atenolol & 619 & & $71 \%$ & 442 \\
Metoprolol tartrate & 2303 & & $71 \%$ & 1641 \\
Carvedilol & 907 & & $75 \%$ & 682 \\
Metoprolol succinate & 1164 & & $67 \%$ & 781 \\
Carvedilol & 907 & & $73 \%$ & 661 \\
Carvedilol & 907 & $63 \%$ & 575 \\
Atenolol & 619 & $59 \%$ & 367 \\
\hline
\end{tabular}

Finding equipoise in a population means that it may be a suitable venue for unbiased research, but without contextual knowledge it may be difficult to distinguish two scenarios that have different implications for the need of additional CER: (1) equipoise may stem from a belief that treatments are interchangeable, which is widely held despite an absence of supporting evidence; (2) equipoise may exist because it is scientifically well established that treatments are indeed equally effective. Only the former scenario indicates a need for more CER.

Table 6 Relative risk of presumed treatment failure in new-onset heart failure in elders

\begin{tabular}{|c|c|c|c|c|c|}
\hline $\begin{array}{l}\text { Beta- } \\
\text { blocker } \\
\text { pair }\end{array}$ & Patients & Failures & $\%$ & $\begin{array}{l}\text { Relative } \\
\text { risk }\end{array}$ & $\begin{array}{l}\text { PS- } \\
\text { adjusted } \\
\text { odds ratio }\end{array}$ \\
\hline $\begin{array}{l}\text { Metoprolol } \\
\text { tartrate }\end{array}$ & 917 & 310 & $16 \%$ & 0.93 & 0.91 \\
\hline Atenolol & 527 & 92 & $17 \%$ & & $(0.70-1.18)$ \\
\hline $\begin{array}{l}\text { Metoprolol } \\
\text { succinate }\end{array}$ & 998 & 138 & $14 \%$ & 0.83 & 0.82 \\
\hline $\begin{array}{l}\text { Metoprolol } \\
\text { tartrate }\end{array}$ & 1983 & 331 & $17 \%$ & & $(0.66-1.03)$ \\
\hline $\begin{array}{l}\text { Metoprolol } \\
\text { succinate }\end{array}$ & 850 & 109 & $13 \%$ & 0.71 & 0.66 \\
\hline Atenolol & 442 & 80 & $18 \%$ & & $(0.48-0.91)$ \\
\hline $\begin{array}{l}\text { Metoprolol } \\
\text { tartrate }\end{array}$ & 1641 & 266 & $16 \%$ & 0.80 & 0.78 \\
\hline Carvedilol & 682 & 138 & $20 \%$ & & $(0.62-0.99)$ \\
\hline $\begin{array}{l}\text { Metoprolol } \\
\text { succinate }\end{array}$ & 781 & 103 & $13 \%$ & 0.68 & 0.61 \\
\hline Carvedilol & 661 & 128 & $19 \%$ & & $(0.45-0.82)$ \\
\hline Carvedilol & 575 & 105 & $18 \%$ & 0.87 & 0.81 \\
\hline Atenolol & 367 & 77 & $21 \%$ & & $(0.57-1.13)$ \\
\hline
\end{tabular}

Note: Patients in each comparison are restricted to those preference scores in the range of 0.3 to 0.7 for that pair.

Data from the Pennsylvania Pharmacy Assistance Contract for the Elderly program, 2000-2005. ${ }^{10}$

Abbreviation: PS, preference score.
Participants in the PACE program are generally female, economically poor, very elderly, and live in Pennsylvania. Would the conclusions of our comparative analyses carry over to young men working in New Mexico? The answer does not lie within the PACE data, but is a matter of scientific generalization. Our speculation is that the individuals whose health events we have assessed here may be different in fundamental ways relevant to drug effectiveness from people who are younger, economically more wealthy, and living in other regions and with differently structured systems of medical care. Accordingly, observational CER needs to be undertaken in many different populations, and the results particularly if they vary - interpreted with care. The goal remains to search out reproducible findings that correspond to generalizable medical facts.

This exercise contrasts with the humorous joke about the inebriated researcher looking for his car keys under a streetlight after a late faculty party. "Did you lose them here?" a colleague asks. "No," comes the reply, "but this is the only spot that I can see anything." To extend the metaphor, what we propose here is a way to map out locations of lights. That comparative research could be done well under a figurative streetlamp (that is, in largely comparable groups) does not mean that it should be done - the addressable issues under the light may be unimportant. However, if we prioritize research according to medical, financial, or societal goals, and as a result find that we would need to undertake non-randomized effectiveness research in the "darkness" of non-comparable groups, we might prefer to take along a flashlight instead: randomization.

The covariates that we included in the preference score calculation were ones that could be derived from insurance claims, but the technique could be applied in any setting in which there is extensive information. If one had an electronic medical record, perhaps supplemented by descriptors of patient lifestyle choices such as smoking, alcohol drinking, or exercise, much more information could have gone into the estimation of whether alternative treatments really were being delivered to substantially similar patients.

PACE, the venue for the examples we have explored, consists of a single social stratum of individuals of relatively homogenous age in a single geographic area, with health care claims data generated under a common reimbursement structure. Observational CER studies that include more heterogeneous data may encounter treatment technologies that vary according to location and other determinants of outcome, not all of them well represented in the claims histories or electronic health records. Depending on the source 
and the time, the data may include fraudulent care, unjustified claims, defective products, and impaired physicians, and may miss addictions or other hidden comorbidities, all to different degrees and possibly differentially associated with the technologies to be compared. Our methods strive to infer equipoise from the comparability of group characteristics in the recipients of different therapy. Social stratification influences who seeks care when, how treatments are delivered, how patients adhere to those treatments, and whether outcomes are even recognized. Observational CER needs to be undertaken in many different populations, and the results particularly if they vary - interpreted with care.

\section{Acknowledgments}

This report is the product of a working group of the Clinical Effectiveness Research Innovation Collaborative. The Collaborative is convened under the auspices of the Institute of Medicine's Roundtable on Value and Science-Driven Health Care to provide a venue for researchers working together to develop and apply innovative approaches to improving the pace and progress of clinical effectiveness research. The work was coordinated and overseen by LeighAnne Olsen, $\mathrm{PhD}$.

Other members of the working group, who contributed to the development of this paper through discussion, review, and constructive suggestions, were Lawrence J Fine and Cato Laurencin. Michael Klompas and Deborah Yokoe reviewed interim versions of this report and provided helpful feedback.

\section{Disclosure}

The authors declare no conflicts of interest in this work.

\section{References}

1. Institute of Medicine Committee on Comparative Effectiveness Research Prioritization. Initial National Priorities for Comparative Effectiveness Research. Washington DC: National Academies Press; 2009. Available from: http://www.nap.edu/catalog.php?record_id=12648. Accessed February 19, 2010.
2. World Health Organization. International Classification of Diseases, 9th Revision - Clinical Modification. Geneva: World Health Organization; 1998.

3. Schneeweiss S, Seeger JD, Maclure M, Wang PS, Avorn J, Glynn RJ. Performance of comorbidity scores to control for confounding in epidemiologic studies using claims data. Am J Epidemiol. 2001;154(9): 854-864.

4. Deyo RA, Cherkin DC, Ciol MA. Adapting a clinical comorbidity index for use with ICD-9-CM administrative databases. J Clin Epidemiol. 1992;45(6):613-619.

5. Mandell LA, Wunderink RG, Anzueto A, et al; Infectious Diseases Society of America; American Thoracic Society. Infectious Diseases Society of America/American Thoracic Society consensus guidelines on the management of community-acquired pneumonia in adults. Clin Inf Dis. 2007;44 Supp1 2:S27-S72.

6. Jessup M,Abraham WT, Chin MH, et al; American College of Cardiology Foundation; American Heart Association. 2009 Focused update incorporated into the ACC/AHA 2005 Guidelines for the Diagnosis and Management of Heart Failure in Adults A Report of the American College of Cardiology Foundation/American Heart Association Task Force on Practice Guidelines Developed in Collaboration With the International Society for Heart and Lung Transplantation. $J$ Am Coll Cardiol. 2009;53(15):e1-e90.

7. Poole-Wilson PA, Swedberg K, Cleland JG, et al; COMET investigators. Comparison of carvedilol and metoprolol on clinical outcomes in patients with chronic heart failure in the Carvedilol Or Metoprolol European Trial (COMET): randomised controlled trial. Lancet. 2003;362(9377): 7-13.

8. Go AS, Yang J, Gurwitz JH, Hsu J, Lane K, Platt R. Comparative effectiveness of different beta-adrenergic antagonists on mortality among adults with heart failure in clinical practice. Arch Intern Med. 2008; 168(22):2415-2421.

9. Kramer JM, Curtis LH, Dupree CS, et al. Comparative effectiveness of beta-blockers in elderly patients with heart failure. Arch Intern Med. 2008;168(22):2422-2428.

10. International Classification of Diseases Clinical Modification (ICD9-CM) October 2011 (CD ROM) US Department of Health and Human Services (DHHS) Publication PHS 11-1260. NCHS CD ROM 2011, No. 1. Available from http://bookstore.gpo.gov/actions/GetPublication. do?stocknumber=017-022-01616-8. Accessed January 8, 2013.
Comparative Effectiveness Research

\section{Publish your work in this journal}

Comparative Effectiveness Research is an international, peer reviewed open access journal focusing on comparative effectiveness of health care including preventative health care strategies, diagnostic strategies, diagnostic technology, medical devices, drugs, medical technology, health systems and organization. The manuscript management system

\section{Dovepress}

is completely online and includes a very quick and fair peer-review system. Visit http://www.dovepress.com/testimonials.php to read real quotes from published authors. 